\title{
GENERAL SURVEYS OF THE BULGE IN THE INFRARED
}

\author{
I.S. GLASS \\ South African Astronomical Observatory, PO Box 9, Observatory 7935, South Africa
}

October 16, 1992

\begin{abstract}
The Bulge has been mapped in the infrared both coarsely from balloon platforms and in detail from the IRAS satellite and the ground. The information revealed by each type of survey is discussed. Particular attention is given to surveys of the Sgr I clear field, its content of long-period variables and their implications for distance determination and the high end of the Bulge luminosity function.
\end{abstract}

Key words: Bulge - surveys - Sgr I

\section{Introduction}

Infrared surveys are particularly important for studies of the Bulge because they are relatively unaffected by the absorption due to the interstellar dust which occupies much of the galactic plane.

More than half of the starlight component is emitted beyond $1 \mu \mathrm{m}$. Photospheres remain the main source of radiation to the end of the $\mathrm{K}$ band $(2.4 \mu \mathrm{m})$, but at longer wavelengths circum- and inter-stellar matter become dominant. By $12 \mu \mathrm{m}$, the shortest IRAS wavelength, photospheric radiation is almost insignificant and even apparently stellar sources are often cool dust shells.

Photography stops at a wavelength of about $1.1 \mu \mathrm{m}$, beyond which electronic detectors are used. Infrared area detectors have small formats at present but are increasing in size; for example at the moment it is possible to work out to $2.2 \mu \mathrm{m}$ ( $\mathrm{K}$ band) with $256 \times 256$ pixels using the NICMOS3 $\mathrm{HgCdTe}$ arrays. InSb arrays of similar size, sensitive up to the $\mathrm{M}$ band $(4.8 \mu \mathrm{m})$, are also starting to become available. PtSi arrays 512 square have been used out to the $\mathrm{K}$ band and others up to 1024 square are under development. Although the PtSi arrays have generally fewer defective pixels than other types, they have only about $5 \%$ quantum efficiency and around $70 \%$ fill factor. Nevertheless, their large formats show promise for largescale survey work.

From the ground, surveys of extended sources at wavelengths longer than the $\mathrm{K}$ band are not possible due to the very high background flux per unit area which overwhelms ordinary area detectors. For point sources it is however becoming feasible to examine larger and larger areas with detectors connected to fast processors which co-add many exposures per second in real time. Observations from space thus have clear advantages for large scale survey work and mapping of extended objects, and are of course a necessity at wavelengths where the earth's atmosphere is opaque.

\section{Infrared Extinction}

Preliminary to the general discussion, it is important to emphasize the behaviour of interstellar extinction in the infrared. The following is a table in general use at 
SAAO, based on the famous van de Hulst (1949) No 15 curve. Some data from Rieke \& Lebofsky (1985) are also shown.

The Rieke \& Lebofsky law, which was based mainly on observations of heavily obscured high-luminosity stars in the direction of the Galactic Centre, differs from the van de Hulst one quite significantly, ie from $15 \%$ higher at $\mathbf{J}$ to $60 \%$ higher at L.

A parameterization of the extinction law of the form $\tau=\mathrm{k} \lambda^{-1.85 \pm 0.05}$, based on comparison of observed to calculated line ratios in an obscured HII region, gives extinction values from the $\mathrm{R}(0.64 \mu \mathrm{m})$ to $\mathrm{M}(4.8 \mu \mathrm{m})$ bands about $10 \%$ lower than the van de Hulst ones (Landini et al, 1984).

TABLE I

Interstellar extinction for common photometric bands.

$\begin{array}{llll}\text { Band } & \lambda & \mathrm{A}_{\lambda} & \mathrm{A}_{\lambda}(\mathrm{R} \& \mathrm{~L}) \\ \mathrm{U} & 0.36 & 1.56 & \\ \mathrm{~B} & 0.44 & 1.33 & \\ \mathrm{~V} & 0.55 & 1.00 & 1.00 \\ \mathrm{R}_{C} & 0.64 & 0.78 & \\ \mathrm{I}_{C} & 0.79 & 0.59 & \\ \mathrm{~J} & 1.25 & 0.245 & 0.282 \\ \mathrm{H} & 1.65 & 0.142 & 0.175 \\ \mathrm{~K} & 2.2 & 0.081 & 0.112 \\ \mathrm{~L} & 3.5 & 0.036 & 0.058 \\ \mathrm{M} & 4.8 & & 0.023 \\ \mathrm{~N} & 10 & & 0.052\end{array}$

The extinction law has been investigated experimentally at SAAO by Glass (unpub.) for A stars and Laney \& Stobie (in prep.) for Cepheids. For example, in the near infrared, the ratio of the colour excess $\mathrm{E}_{B-V}$ to $\mathrm{E}_{J-H}$ and $\mathrm{E}_{H-K}$ are found to agree with the van de Hulst law to within a few percent. However, $\mathrm{E}_{B-V} / \mathrm{E}_{V-K}$ is only known to about $1 \%$ which means that the ratio of $A_{K}$ to $A_{V}$ is known to $10 \%$. At longer wavelengths the determination of extinction becomes more difficult because the values are so small and "infrared excesses" become more common. It is always important to remember that different groups use infrared filters which differ slightly in effective wavelength from one to another and that slightly different ratios will therefore apply.

\section{Low-resolution studies of the Bulge}

Absolutely calibrated large-scale observations of the Bulge are difficult from the ground because of atmospheric absorption and emission, which complicate the determination of background levels. Apart from ordinary thermal radiation which becomes prominent around $2.4 \mu \mathrm{m}$, there is rapidly variable emission from $\mathrm{OH}$ high $(\sim 90 \mathrm{~km})$ in the atmosphere (airglow). For this reason, such work has traditionally 
been carried out from balloon-borne platforms in carefully chosen bands and more recently from space vehicles such as the Shuttle and COBE.

The existing surveys are summarized in the Table II

TABLE II

Large area maps of the bulge at low resolution.

\begin{tabular}{|c|c|c|c|c|c|}
\hline Group & Resolution & $\lambda(\mu m)$ & $\Delta \lambda$ & Range in $l$ & Reference \\
\hline Kyoto & $1^{\circ} \mathrm{sq}$ & 2.4 & .09 & $-10^{\circ}$ to $+30^{\circ}$ & $\begin{array}{l}\text { Okuda et al (1977), } \\
\text { Maihara et al }(1978)\end{array}$ \\
\hline Nagoya & $2^{\circ} \mathrm{sq}$ & 2.4 & .1 & $-10^{\circ}$ to $+30^{\circ}$ & Ito et al (1977) \\
\hline \multirow[t]{2}{*}{ MPIA } & $2^{\circ}$ diam & 2.4 & .15 & $0^{\circ}$ to $+10^{\circ}$ & Hofman et al (1977) \\
\hline & & 3.4 & .2 & & \\
\hline MPIA & $1^{\circ} \mathrm{sq}$ & 2.45 & .09 & $-7^{\circ}$ to $+7^{\circ}$ & Hofman et al (1978) \\
\hline \multirow[t]{2}{*}{ Nagoya ++} & $1.7^{\circ} \mathrm{sq}$ & 2.4 & .09 & $-70^{\circ}$ to $+50^{\circ}$ & Hayakawa et al (1981) \\
\hline & & 3.4 & .14 & & \\
\hline Kyoto & $0.6^{\circ} \mathrm{sq}$ & 2.38 & .08 & $-15^{\circ}$ to $+33^{\circ}$ & Oda et al (1979) \\
\hline \multirow[t]{2}{*}{ Nagoya ++} & $0.5^{\circ}$ diam & 2.4 & .09 & $-12^{\circ}$ to $+12^{\circ}$ & Matsumoto et al (1982) \\
\hline & $0.7^{\circ}$ diam & 3.4 & .14 & & \\
\hline Kyoto & $0.4^{\circ}$ diam & 2.4 & .08 & $-5^{\circ}$ to $+5^{\circ}$ & Hiromoto et al (1984) \\
\hline Harv-Smith & $\sim 1^{\circ}$ & 2.4 & 1.3 & $-8^{\circ}$ to $+100^{\circ}$ & Kent et al (1992) \\
\hline COBE & $0.7^{\circ} \mathrm{sq}$ & JKLM & & $-96^{\circ}$ to $+96^{\circ}$ & NASA photograph $(1990$ \\
\hline
\end{tabular}

The last map is presented as a three-colour photograph combining the $\mathrm{J}$ (1.25), $\mathrm{K}(2.2)$ and $\mathrm{L}(3.4 \mu \mathrm{m})$ images obtained by the DIRBE (Diffuse Infrared Background Experiment) on the COBE (Cosmic Background Explorer). No quantitative results of this experiment have been released at the time of writing.

\subsection{INTERPRETATION}

The Nagoya group (Matsumoto et al, 1982) data at $0.5^{\circ}$ resolution are the most refined of the large-scale surveys and they have been analysed by Blitz \& Spergel (1991) who searched for a bar which appears from dynamical considerations to be affecting the gas kinematics in the central few kpc of the Galaxy. The somewhat bar-shaped bulge revealed by COBE and the Nagoya data have been identified with the hypothesized bar and analysed for non-axisymmetric properties in the hope of revealing its orientation to the line of sight. It is assumed that at galactic latitudes beyond $\pm 3^{\circ}$ the data are unaffected by reddening. They placed the data in $1^{\circ}$ bins and subtracted each bin at "negative" longitude from its counterpart at positive longitude to form a difference map which clearly shows an excess at positive longitudes, i.e. the near end of the bar is at positive longitudes and appears wider. Further, the bar appears to be tilted so that there is slightly more flux at negative $b$ and positive $l$ with less at positive $b$ and "negative" $l$.

To be certain that the measured effects are not consequences of uneven extinction, they appeal to the Milky Way CO maps of Dame et al (1987) and the 
well-known correlation of dust and gas distributions. If anything, there is slightly more $\mathrm{CO}$ at positive than negative longitudes, mainly in a narrow plume, which would work against rather than for the longitudinal asymmetry. In general, the maximum visual extinction in the region examined is estimated to be less than 1.3 mag, corresponding to less than $0.13 \mathrm{mag}$ at $2.4 \mu \mathrm{m}$. The asymmetry noted is of order $15 \%-40 \%$, considerably more than any possible differential extinction could account for.

We may compare the above results with those of Binney et al (1991) who considered a particle in a bar-like potential arising from a prolate ellipsoid of axis ratio 0.75 and mass density varying as $\mathrm{r}^{-1.75}$. Its orbits were compared with the parallelogram-like ${ }^{12} \mathrm{CO}$ (longitude, velocity) diagram for $|b|<0.1^{\circ}$. They concluded that the bar's axis should be at about $16^{\circ}$ to the line of sight and co-rotation would occur at $2.4 \mathrm{kpc}$. The pattern speed of the bar is $81 \mathrm{~km} \mathrm{~s}^{-1} \mathrm{kpc}^{-1}$ from the dynamics of the gas which is similar to the mean velocities of planetary nebulae as a function of galactic longitude (Kinman et al, 1988). Mira variables at b $7^{\circ}$ (Menzies, 1990) also rotate at these speeds.

\section{Surveys with high resolution}

The IRAS satellite as is well known, surveyed the Bulge with sufficient resolution to pick up individual objects.

However, it is also known that the high source density in the galactic plane led to incomplete surveys, or 'shadows', in the regions $l=10^{\circ}$ to $30^{\circ}, b=0^{\circ}$ to $5^{\circ}$ and $l=330^{\circ}$ to $350^{\circ}, b=0^{\circ}$ to $-5^{\circ}$, especially at the longer wavelengths. Fig VIII.D.2 of the IRAS Explanatory Supplement (Beichman et al, 1988) shows that possibly about $1 / 3$ of the 12 micron sources within $1^{\circ}$ of the plane, in the longitude range $10^{\circ}$ to $30^{\circ}$, were not detected. The effects of crowding and saturation are worse nearer to the Galactic Centre and become more severe at longer wavelengths.

It was quickly realised by Habing (1985) that the distribution of IRAS sources with $12 \mu$ m fluxes less than $5 \mathrm{Jy}$ and comparable $25 \mu \mathrm{m}$ fluxes very much resembles the view of many edge-on spiral galaxies, showing a small bulge and a disc. Feast (1985) showed that many of the IRAS sources in the Sgr I and NGC6522 fields of the Bulge could be identified with known mira variables. Criteria were devised by van der Veen \& Habing (1988) in terms of the [12]-[25], [25]-[60] two colour diagram (Fig 1) in order to separate objects according to degree of dominance by circumstellar shells, interpreted by them in evolutionary terms. The shells associated with O-rich variables have temperatures of around 200-300K.

The spatial distribution of sources in the category IIIb (thick dust shells) is particularly concentrated towards the Bulge. Thirty-seven IIIb bulge candidates were observed a number of times in the KLMN and the ESO narrow N band filters $(2.2$ to $10 \mu \mathrm{m})$ and most were shown to have silicate absorption features, indicative of optically thick dust shells as expected (van der Veen \& Habing, 1990). Periods of 500 to 1990 days were derived for these stars. Some of the longer period stars were undersampled in phase and have been since been revised to have shorter periods (van der Veen \& Habing, in preparation). More than $50 \%$ of the sample are identified with OH/IR stars from the te Lintel Hekkert et al (1990) survey. Blommaert et al 


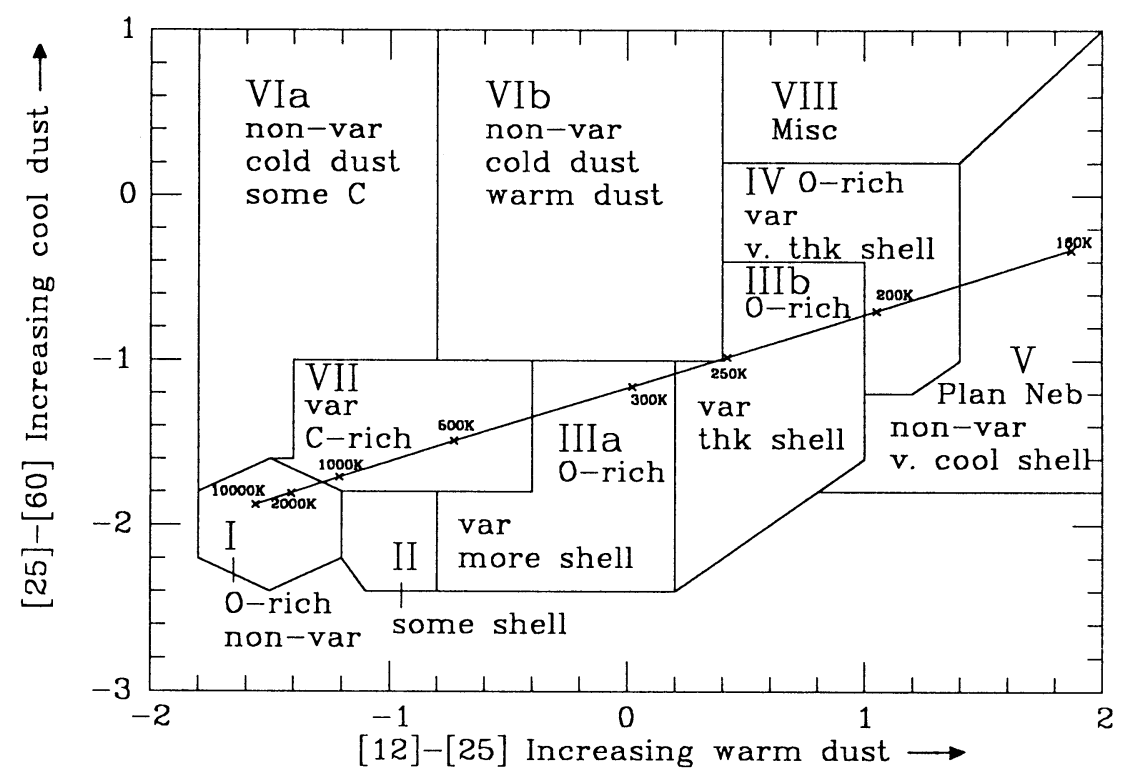

Fig. 1. Regions in the IRAS two-colour diagram which denote various types of stars with dusty envelopes (van der Veen \& Habing, 1988).

(1992) report on a similar survey starting from OH/IR stars from a field within $30^{\prime}$ of the Centre which appears to show that they are closely related to the van der Veen \& Habing stars and also that their bolometric magnitudess are below the (extrapolated) LMC P-L relation for M-type miras.

\subsection{IRAS AND THE BAR}

More evidence for the bar-like structure of the Bulge has come from analysis of IRAS data. Early work on the distribution of Bulge sources by Harmon \& Gilmore (1988) showed a slight asymmetry. Nakada et al (1991) analysed a sample of sources away from the Plane, having a narrow range of colours on the border of IIIa and IIIb and assuming $M_{B o l} \sim$ constant. They found evidence that the sources in the first quadrant are nearer the sun.

Almost simultaneously, Weinberg (1992a) made a similar analysis by selecting variable sources away from the Plane and having [12]-[25] colours in the reddest quartile (presumably mostly of type IIIb), assuming constant $\mathrm{M}_{B o l}$ and calculating photometric distances using $\mathrm{M}_{B o l}$ estimated for each star from its IRAS fluxes. A map of source numbers with inferred position in the galactic plane for $\mathrm{F}_{12}>2 \mathrm{Jy}$ just reveals the Galactic Centre with a bar in the 1st quadrant. Going to $\mathrm{F}_{12}>1$ Jy begins to show the other end of the bar. The semimajor axis of the bar is $5 \mathrm{Kpc}$ and the tilt to the line of sight $36^{\circ}$.

In a more recent paper, Weinberg $(1992 \mathrm{~b})$ has carried his analysis of the spatial distribution of the variable $\mathrm{F}_{12}>2 \mathrm{Jy}$ sources somewhat further and finds that 
the entire distribution is best explained by a single exponential disk distribution with $\mathrm{a}=4.5 \mathrm{kpc}$ and $\mathrm{h} \sim 800 \mathrm{pc}$. Any $\mathrm{r}^{1 / 4}$-type Bulge component is quite small. Weinberg's choice of minimum $12 \mu \mathrm{m}$ flux is such that about half the IRAS sources in the Sgr I window are excluded. Thus only the most luminous variables of the Bulge contribute to his sample. These are the longest period sources, which are likely to belong to the most flattened (old disc) component of the long period variable population (see e.g. Feast, Whitelock \& Sharples, 1992).

\subsection{Near-Infrared Surveys and their Follow-UP}

The nearest infrared surveys, conducted by Blanco and collaborators in the I band $(\sim 0.8 \mu \mathrm{m})$, are capable of showing Bulge stars in the "clear" windows of low extinction. However, as pointed out by Frogel (1988), the brightest stars at I are not necessarily the most luminous bolometrically because of line blanketing effects. Photographic surveys are however very useful in that large areas can be covered in a short time.

Objective prism surveys, also by Blanco and collaborators, have been used for spectral classification of Bulge stars in the clear windows on a large scale. Some confusion has entered the literature due to the fact that the Blanco classifications were not exactly according to the Morgan-Keenan standard system, as pointed out by Terndrup et al (1990). In effect, the M stars of subtypes between M0 and M7 were classified too late by one to two subtypes.

Frogel \& Whitford (1987) showed that Bulge stars have bluer J-K and H-K colours than local giants of the same spectral class, and this conclusion remains in spite of the revision of the objective prism spectral types. However one should remember that for stars of high metallicity, the bands used for classification purposes are more pronounced, independent of luminosity and temperature, and thus to some extent spectral type becomes confusing, especially when discussing luminosities.

Independently of spectral type, the mean locus in the $\mathrm{J}-\mathrm{H}, \mathrm{H}-\mathrm{K}$ diagram shows a progression from left to right as one goes from globular clusters to local giants to Bulge giants (Frogel, Terndrup, Blanco \& Whitford, 1990). This is a powerful indicator of metallicity differences, although there are some "local" giants at a mean distance of $500 \mathrm{pc}$ from the Plane with colours similar to those in the Bulge (Feast, Whitelock \& Carter, 1990).

In the colour-magnitude diagram, an interesting characteristic is that the luminosities of Bulge $M$ stars extend to half a magnitude greater than those in globular clusters. Frogel (1988) points out that the K, J-K diagram does not change significantly with latitude, and this suggests that the effect noted in the J-H, H-K diagram occurs mainly in the $\mathrm{H}$ band. $\mathrm{J}-\mathrm{K}$ is a good indicator of temperature, independent of metallicity (Terndrup, Frogel \& Whitford, 1991).

It is often remarked that the Bulge giant branch is quite broad and therefore indicative of a spread in metallicity. Little account seems to have been taken of the spread caused by the different distances of Bulge stars in the line of sight, which will of course become larger as distance from the Centre increases. Indications from mira work are that a spread of magnitudes should be expected, having a FWHM of about 0.5 to $0.7 \mathrm{mag}$ in the $\mathrm{Sgr}$ I field at $\sim 3^{\circ}$ from the Centre. The line of sight 
depth will also be affected by the barred nature of the Bulge.

The choice of late-type M-stars by Frogel and co-workers for discussion of metallicity questions may give a distorted view of the population as a whole. It is wellknown that a population that is metal-rich will allow evolution to later spectral types than one which is metal-poor. Thus, in a population containing stars which cover a large metallicity range, it is likely that the late $\mathrm{M}$ stars will be part of the metal rich fraction of the general distribution. $\mathrm{K}$ giants may be more representative of average metallicity, since all stars go through a $\mathrm{K}$ giant phase. A sample of 88 Bulge giants observed by Rich (1988) shows about that about $50 \%$ exceed solar metal abundance and $20 \%$ are super metal rich.

\subsection{K-BAND SuRveys}

K-band-infrared surveys provide a more objective way of picking out the true highluminosity late-type stars from a population because of the monotonic relation between $\mathrm{K}$ mag and $\mathrm{m}_{\mathrm{Bol}}$ for giants (excepting those possessing very thick shells).

So far, K surveys have covered relatively small areas. The largest JHK survey of the bulge that is sensitive enough to show individual stars remains that of the SAAO group, covering an area of $2^{\circ} \times 1^{\circ}$ in RA and Dec. It stretches from just west of the G.C. to about $1.5^{\circ}$ east of it. Qualitatively (Glass, Catchpole \& Whitelock, 1987) it shows that even at $2.2 \mu \mathrm{m}$ wavelength there remain considerable patches of extinction $\left(\mathrm{A}_{V}>60 \mathrm{mag}\right)$. This extinction is strongly correlated with low-velocity molecular gas, presumed to be in circular orbits in the foreground. The vast column densities of high velocity gas revealed by the $\mathrm{CO}$ surveys are not correlated with readily visible features in the extinction and if there is dust associated with this material in the usual proportions its location must be behind the Centre.

One of the unexpected results of the SAAO survey was a very marked increase in the number of stars of high luminosity $\left(5<\mathrm{K}<6\right.$ or $\left.\mathrm{M}_{B o l} \sim-5\right)$ towards the Centre (Fig 2). These have a flattened distribution lying along the longitude axis, but are very much peaked towards the Centre even along that axis. Haller \& Rieke (1989) have independently found a population of luminous M stars within a few arcmin of the Galactic Centre. These objects do not appear to vary and are believed to owe their high luminosity to relative youth. Evidence for recent star formation near the Centre and mostly close to the Galactic Plane has been presented by Moneti, Glass \& Moorwood (1992). The IRAS maps of unresolved continuum emission (presumably due to dust) presented by Cox \& Laureijs (1989) also suggest the possibility of ongoing star formation.

The quantitative results of the SAAO survey are presented by Catchpole, Whitelock \& Glass (1990). Stars from sub-fields of $400^{\prime \prime}$ by $400^{\prime \prime}$ were plotted on colourmagnitude $(\mathrm{H}-\mathrm{K}, \mathrm{K})$ diagrams. Assuming that most of the stars were late-type giants and that they possessed the colours of giants from other fields, the average reddening for each sub-field could be found and the true stellar distribution in the plane of the sky at $\mathrm{K}$ could be determined.

A composite $\mathrm{H}$ and $\mathrm{K}^{\prime}(2.15 \mu \mathrm{m})$ picture of the central part $\left(3.6^{\circ} \mathrm{E}-\mathrm{W}\right.$ by $3.6^{\circ}$ $\mathrm{N}-\mathrm{S}$ ) of the galaxy has been made by combining 35 fields observed with the $512 \mathrm{x}$ 512 PtSi-based infrared camera of the National Observatory of Japan by Munetaka 


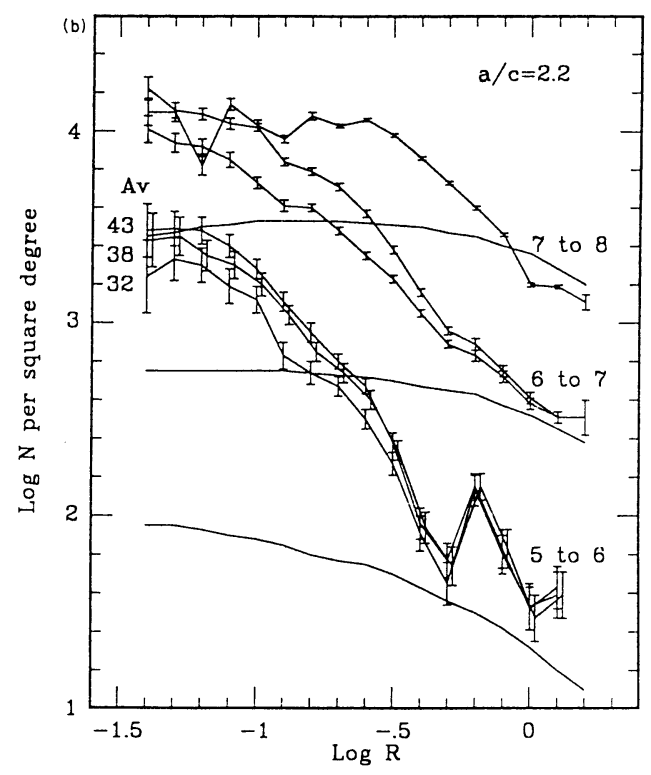

Fig. 2. No of stars per square degree in various (dereddened) $\mathrm{K}$ magnitude intervals shown as a function of $\log \mathrm{R}$ (degrees from the Centre) for elliptical annuli having $\mathrm{a} / \mathrm{c}=2.2$, corrected for crowding, interstellar absorbtion and contamination by Disc stars. The continuous curves show the Disc numbers that have been subtracted. The $A_{V}$ numbers represent the $A_{V}$ out to which counts have been made. For the $7<\mathrm{K}<8$ curve, the upper limit of $A_{V}$ was $32 \mathrm{mag}$. From Catchpole, Whitelock \& Glass (1990)

Ueno (private communic'ation) of the University of Tokyo. The resolution is about 10 arcsec. Each field was exposed twice for 240s with a small shift between exposures. The limiting mags are 12.0 at $\mathrm{K}^{\prime}$ and 13.0 at $\mathrm{H}$.

\subsubsection{The Sgr I field}

The Sgr I field, lying at $l=1.4^{\circ}, b=-2.6^{\circ}$, is one of those clear fields surveyed at B,V \& I for long-period variables by Lloyd Evans (1976) and others. A portion (205 $\operatorname{arcmin}^{2}$ ) of this field (Catchpole, Glass \& Whitelock, unpublished) was scanned in $\mathrm{J}, \mathrm{H} \& \mathrm{~K}$ at the time of the $1^{\circ}$ by $2^{\circ}$ survey referred to above (see Fig 3 ). Sixteen mira variables were identified in the scanned area, of which five of the reddest and brightest were detected by IRAS. From their agreement with the P-L-C relation these miras are clearly part of the Bulge and not of the foreground. The non-miras cannot be so easily placed as to distance. Some objects to the blue of the giant branch are probably foreground. Walker, Sharples \& Cropper (1990) found from a kinematic survey of 225 stars in the NGC 6522 window that the brightest stars at I in their sample have systematically lower dispersion than the others, suggesting that they are foreground (disc) objects.

The 19 IRAS sources in the whole $1200 \operatorname{arcmin}^{2} \mathrm{Sgr}$ I field of Lloyd Evans 


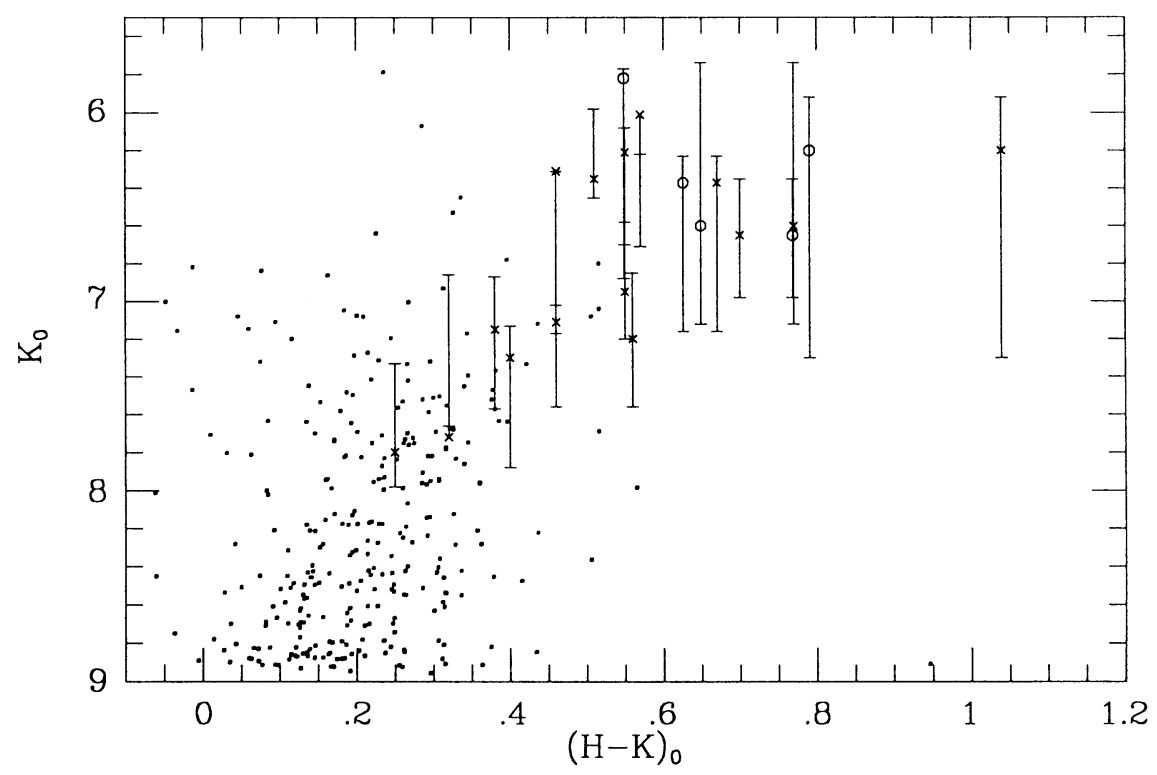

Fig. 3. $\mathrm{K}_{0}$ vs $(\mathrm{H}-\mathrm{K})_{0}$ for a $205 \mathrm{arcmin}^{2}$ portion of the Sgr I clear field from the SAAO JHK survey of the central Bulge. Mira variables which were found by Lloyd Evans (1976) or previous workers are marked with crosses. The bars represent the magnitude limits of the variables as observed during a programme of photometry of individual stars (Glass, Whitelock, Catchpole \& Feast, in preparation). Colours should be accurate to about \pm 0.1 mag.

(1976) have been found to be mira or mira-like variables with only two exceptions. A programme of repeated observations of these and other known mira variables, expanding on the photometry by Glass \& Feast (1982), has been completed recently (Glass, Whitelock, Catchpole \& Feast, in preparation) to determine better periods, magnitudes and colours. The Sgr I miras fall slightly below the P-L relation (see Fig 4) but follow closely the period-luminosity-colour relation established for Large Magellanic Cloud M-type miras by Feast et al (1989).

The miras, because of their adherence to a period-luminosity relation, are clearly identifiable as members of the Bulge, with a few possible exceptions. This implies that their true luminosity function can be established, without fear of contamination by foreground stars.

The distance to the Centre can be obtained from the photometrically determined distances of individual mira variables (Glass \& Feast, 1982). We are now using the LMC P-L-C relation to repeat the determination. A prelimary analysis of the improved data, based on modelling the mira distribution as a function of distance from the Centre, an ellipsoidal Bulge, an assumed power-law stellar density distribution and a particular value for the combined intrinsic and observational scatter has been performed (Fig 5). The distance itself is not particularly sensitive to the modelling parameters. (Discussed further by Whitelock, these proceedings.) 


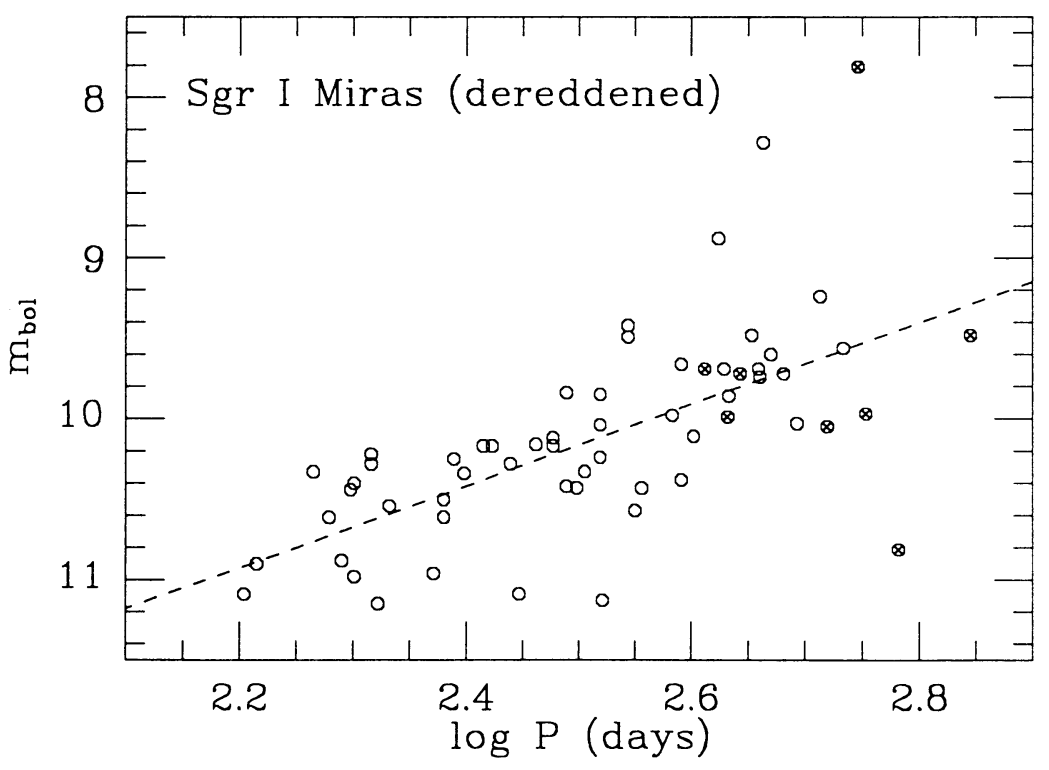

Fig. 4. The average bolometric mags of miras in the Sgr I field as a function of period. (from Glass, Whitelock, Catchpole \& Feast, in preparation). The best fit P-L relation, omitting the two (apparently) most luminous sources, is shown, viz $\mathrm{m}_{B o l}=-2.54 \log \mathrm{P}$ +16.53 . The scatter in this diagram is partly due to the depth of the field in the line of sight, the colour dependence of $\mathrm{M}_{B o l}$, the observational errors and the actual intrinsic scatter. (The filled circles make use of IRAS data)
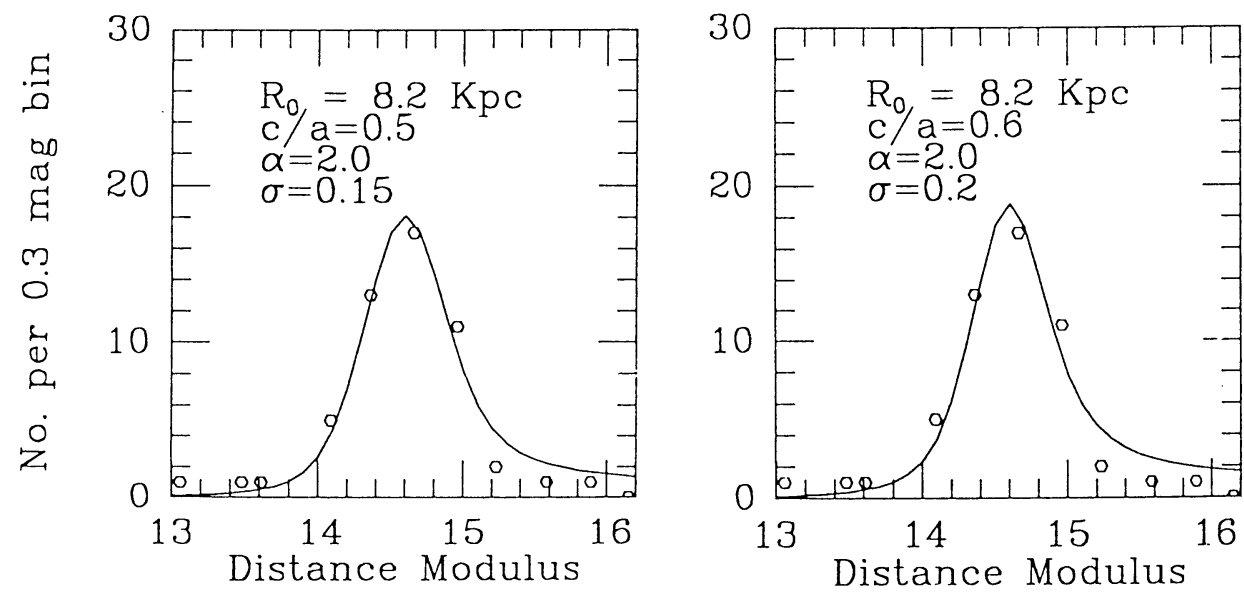

Fig. 5. Two examples of the fit to the distance distribution of the Sgr I miras from their $\mathrm{M}_{B o l}$ determined from the P-L-C relationship. $\mathrm{c} / \mathrm{a}$ is the axial ratio of the model assumed for the ellipsoidal Bulge and $\alpha$ is the exponent of the power law density distribution assumed for mira variables. $\sigma$ is the assumed combined intrinsic and observational scatter. 


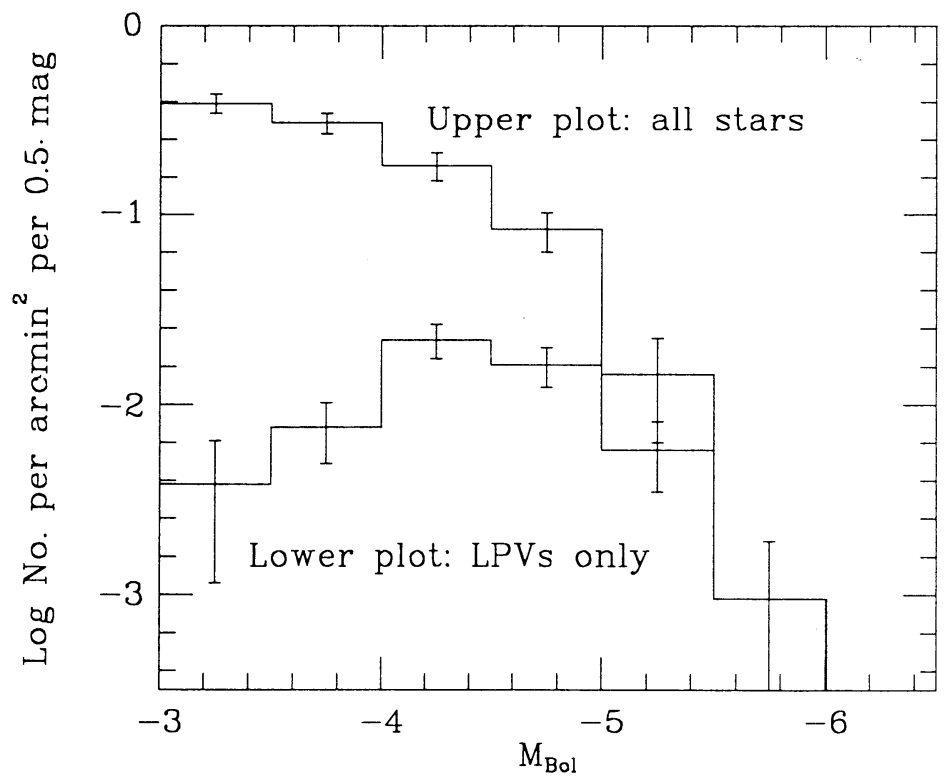

Fig. 6. Apparent luminosity function (not corrected for distance spread) for the Sgr I field based on multi-phase photometry of miras and the $\mathrm{K}$ scans mentioned in the text. The bolometric magnitudes of the non-miras have been formed simply by adding 3.1 to their de-reddened $\mathrm{K}$ mags. The tail-end of the luminosity function is comparable with that for NGC 6522 found by Frogel \& Whitford (1987). Although Frogel \& Whitford's cut-off $\mathrm{M}_{B o l}$ is usually given as $\sim-4.9$ (if the distance modulus $=14.6$ ), there are a few LPVs in each field which extend the distributions to an average $\mathrm{M}_{B o l}$ of $\sim-5.5$. The two apparently most luminous stars of Fig 4 have been omitted as they probably lie in the foreground.

The luminosity function of the mira variables in the Sgr I field can be determined quite accurately because the distances of the miras are known and we do not need to subtract the numbers of foreground stars according to some model (Fig 6).

It is clearly interesting to know how mira variables can be detected most efficiently in the Bulge. Fig. 7 shows the success of the various techniques. The blue survey was conducted by Oosterhoff \& Ponsen (1968), that in the V and I band by Lloyd Evans (1976) and the IRAS results are taken from the Point Source Catalog, regardless of detection quality (Glass, 1986). Clearly none of these surveys were complete for all period ranges. The Lloyd Evans technique (Lloyd Evans, private communication) probably yields completeness to about 300 days. There is a possibility that some of the longer-period variables were not detected due to faintness at I and diminished IRAS sensitivity near the Galactic Plane. It appears that a $2.2 \mu \mathrm{m}$ survey of rather modest depth but large area would be ideal for this sort of work. It would offer the possibility of mapping the reddening from mira colours in considerable spatial detail. The relationship between mira kinematics and period for local miras and the period distribution of miras in globular clusters also suggests a strong connection between age (or perhaps metallicity) and period for miras which 


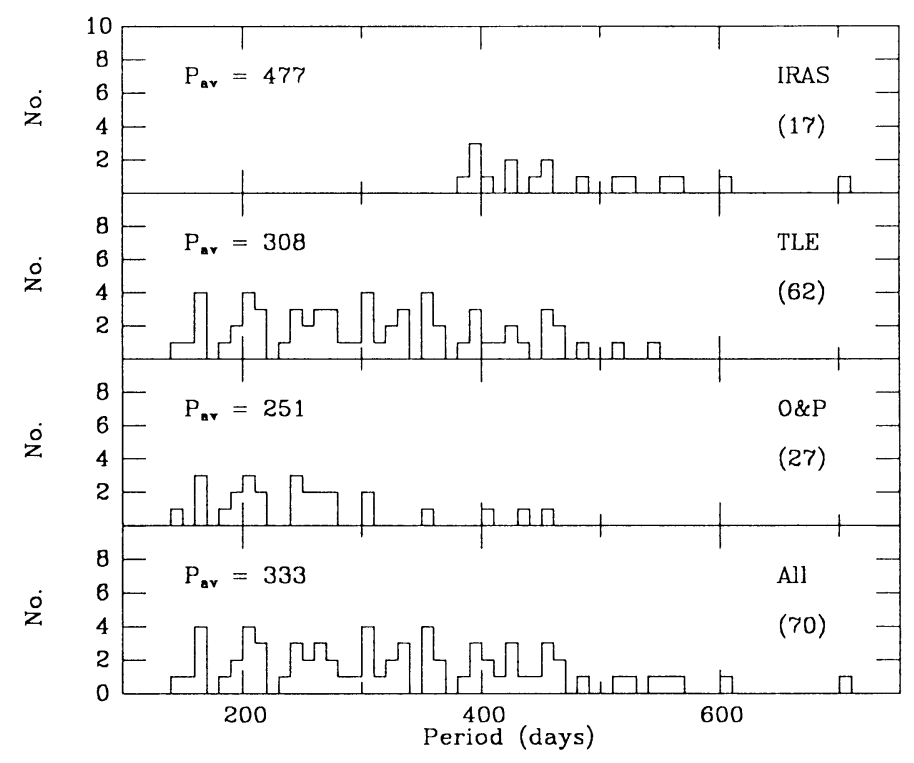

Fig. 7. Histograms of the miras in the Sgr I field according to method of detection.

could be of use in exploring the formation of the Bulge.

The average J-H, H-K colours of the Sgr I miras follow those of the solar neighbourhood rather than the LMC, at least for periods of up to about 300 days. Beyond 300 days, they are distinctly redder than the other samples, as previously noted by Wood \& Bessell (1983). If these results are interpreted in terms of metallicity, we can say that the short period miras of the Bulge and solar neighbourhood and Galactic Centre are similar to each other but more metal rich than their LMC counterparts, while the longer period miras in the Bulge are more metal rich than those known in the solar neighbourhood and are not represented at all in the LMC. However, the solar neighbourhood sample is mainly based on visual and blue surveys and our knowledge of the long-period end is thus distorted. The one population difference that is certain is the existence of carbon miras locally and in the LMC and their complete absence in the Bulge.

\subsubsection{The NGC 6522 field}

Part of the NGC 6522 field $\left(201 \operatorname{arcmin}^{2}\right.$ ) has been scanned at $2.2 \mu \mathrm{m}$ by RuelasMayorga \& Teague (1992). This was extended to fainter magnitudes by scanning a smaller area with a larger telescope. The brightest 165 objects found in the scans were re-observed in a programme of discrete photometry. From this work they constructed a Cumulative Counts Function (integrated K luminosity function) for the NGC 6522 window. In an application of the NICMOS3 near-infrared array, Davidge (1991) has examined a $1.6 \mathrm{arcmin}^{2}$ area of the NGC 6522 field. This work is compared to that of Ruelas-Mayorga \& Teague (1992) in Fig.9. The agreement 


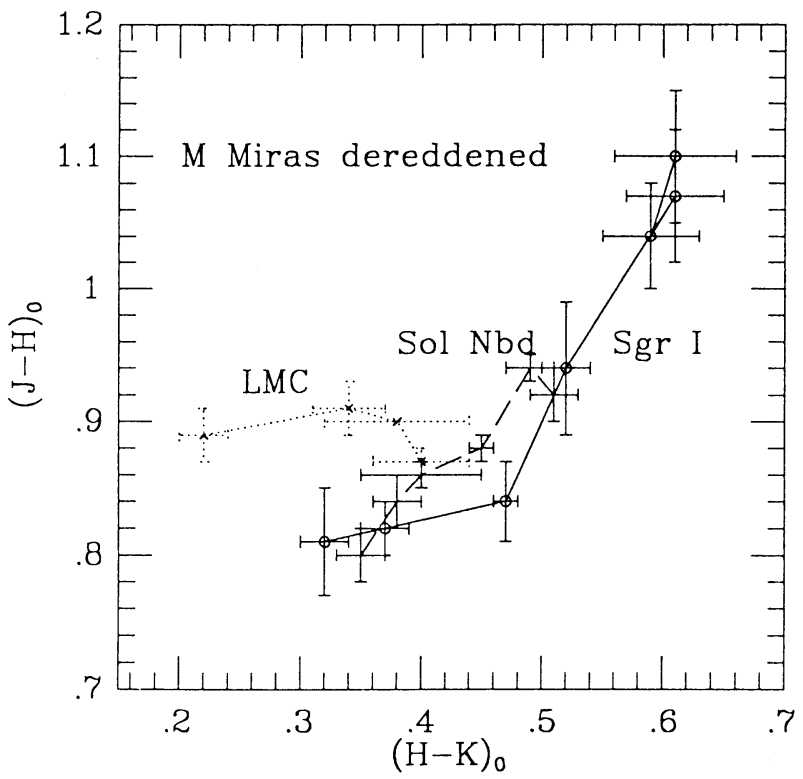

Fig. 8. Summary J-H, H-K diagram for LMC, local (O-rich) and Sgr I miras.

is fairly satisfactory. This field and also the Sgr II field have been surveyed for mira variables at I by Lloyd Evans (1976). Many of the miras in NGC 6522 were observed repeatedly by Glass \& Feast (1982) and once by Wood \& Bessell (1983).

\subsubsection{Other fields that have been surveyed}

Ruelas-Mayorga \& Teague (1992, in press) have made scans of three small fields (56 to $185 \mathrm{arcmin}^{2}$ ) along the latitude axis of the galaxy, at latitudes of $3.5^{\circ}, 4^{\circ}$ and $5^{\circ}$.

\subsubsection{Outer Bulge}

Multi-phase photometry of IRAS sources in two fields lying at $7^{\circ}<|b|<8^{\circ}$, $345^{\circ}<l<15^{\circ}$, to determine periods and average magnitudes, has been reported by Whitelock, Feast \& Catchpole (1991). One of the fields partially overlaps Plaut field No 3 , at $l=0^{\circ}, b=-10^{\circ}$, which has been surveyed using $\mathrm{B}$ and R photographic plates for miras and other variable stars by Wesselink (1987). Although our knowledge of mira variables at intermediate periods is likely to be uncertain in this field, there is a strong suggestion that the numbers of very long-period variables that IRAS detects compared to those of short period is greater in the Sgr I field than in the $-10^{\circ}$ degree field (see contribution by P.A. Whitelock), again a suggestion of a metallicity gradient in the mira population. The SAAO photometric observations of IRAS sources have turned up very few miras of period exceeding 700 days, although other authors claim to have found longer period sources on the basis of 


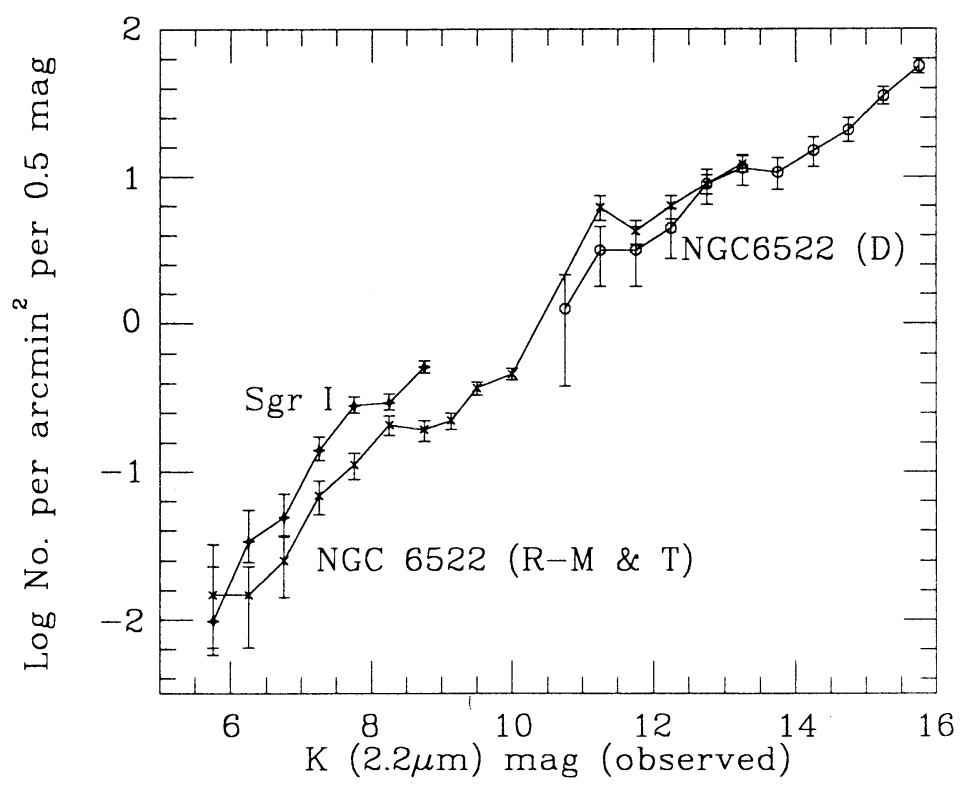

Fig. 9. The luminosity function of the NGC 6522 field at $\mathrm{K}$ as determined from data obtained by Ruelas-Mayorga \& Teague (1992) and Davidge (1992). The luminosity function from the unpublished SAAO scans of Sgr I is also shown. All data are presented as observed, ie without any correction for interstellar absorption (which increases $\mathrm{K}$ by $\sim 0.15$ ). About $30 \%$ of the stars brighter than $\mathrm{K}=6.7$ are non-miras and thus possibly foreground).

sparser observations. Only in two cases has direct evidence been found for an incorrect period (Whitelock et al, 1991), but the suspicion remains that many of the long periods claimed or hypothesized by interpreters of IRAS data, for example Harmon \& Gilmore (1988), are not realistic. An analysis of the bolometric mags derived from this programme by Whitelock \& Catchpole (1992) and using the P-L relationship to obtain distances, shows that there is a marked longitude asymmetry which can be modelled by a prolate spheroidal stellar distribution having $\mathrm{x}_{0}=760$ $\mathrm{pc}, \mathrm{y}_{0}=\mathrm{z}_{0}=190 \mathrm{pc}$ with an angle of inclination to the line of sight of $45^{\circ}$, lending further weight to the bar hypothesis.

\section{Extension to other galaxies}

Surveys of the bulge of M31, which might be expected to be similar to that of the Milky Way, have been conducted by Rich \& Mould (1991) and Davies, Frogel \& Terndrup (1991). The first of these papers finds that the luminosity function in M31 extends considerably beyond Frogel \& Whitford's cut off, by almost 1 mag. However, taking the distance modulus to the Centre as 14.6, the difference is hardly significant and amounts to an argument about small-number statistics. The Davies et al paper finds a similar result, but the suggestion is made that the M31 bulge sample is contaminated by luminous disc stars from a younger population. Studies 
of the luminosity function in other galaxies less similar to ours show more convincing evidence for extensive populations of higher luminosity stars than are met with in the Bulge. For example, Freedman (1992) finds a highly luminous group of latetype stars (including many non-variables) in the dwarf elliptical M32, which she suggests are from an intermediate age population. Similarly, and perhaps more expectedly, Mould (1992) finds intermediate age populations of high luminosity in the Magellanic Clouds.

\section{Acknowledgements}

I thank R.M. Catchpole, M.W. Feast \& P.A. Whitelock for permission to quote unpublished material from joint programmes concerning the Sgr I region. P.A. Whitelock and T. Lloyd Evans are thanked for critical readings of a draft of this article.

\section{References}

Beichman, C.A., Neugebauer, G., Habing, H.J., Clegg, P.E. \& Chester, T.J., 1988. IRAS Explanatory Supplement, NASA RP-1190, Washington, D.C.

Binney, J., Gerhard, O.E., Stark, A.A., Bally, J. \& Uchida, K.I., 1991. MNRAS, 252, 210.

Blitz, L. \& Spergel, D.N., 1991. Ap J, 379, 631.

Blommaert, J.A.D.L., van Langevelde, H.J., Habing, H.J., van der Veen, W.E.C.J. \& Epchtein, N., 1992. In Warner, B., (ed) Variable Stars \& Galaxies; Symposium in Honour of M.W. Feast, A.S.P. Conf. Ser., San Franscisco.

Catchpole, R.M., Whitelock, P.A. \& Glass, I.S., 1990. MNRAS, 247, 479.

Cox, P. \& Laureijs, R., 1989. In Morris, M., (ed) IAU Symp. 136, The Centre of the Galaxy, Reidel, Dordrecht.

Dame, T.M., Ungerechts, H., Cohen, R.S., de Geus, E.J., Grenier, I.A., May, J., Murphy, D.C., Nyman, L.-A. \& Thaddeus, P., 1987. Ap J, 322, 706.

Davidge, T.J., 1991. Ap J, 380, 116.

Davies, R.L., Frogel, J.A. \& Terndrup, D.M., 1991. AJ, 102, 1729.

Feast, M.W., 1985. In Israel, F.P., (ed) Light on Dark Matter, Reidel, Dordrecht.

Feast, M.W., Glass, I.S., Whitelock, P.A. \& Catchpole, R.M., 1989. MNRAS, 241, 375.

Feast, M.W., Whitelock, P.A., \& Carter, B.S., 1990. MNRAS, 247, 227.

Feast, M.W., Whitelock, P.A. \& Sharples, R., 1992. In Barbuy, B. \& Renzini, A., (eds) IAU Symposium 149: The Stellar Populations of Galaxies, Reidel, Dordrecht.

Freedman, W.L., 1992. $A J$, (in press).

Frogel, J.A., 1988. Ann Rev A Ap, 26, 51.

Frogel, J.A., Terndrup, D.M., Blanco, V.M. \& Whitford, A.E., 1990. Ap J, 353, 494.

Frogel, J.A. \& Whitford, A.E., 1987. Ap J, 320, 199.

Glass, I.S., 1986. MNRAS, 221, 879.

Glass, I.S., Catchpole, R.M. \& Whitelock, P.A., 1987. MNRAS, 227, 373.

Glass, I.S. \& Feast, M.W., 1982. MNRAS, 198, 199.

Habing, H.J., 1985. In Israel, F.P., (ed) Light on Dark Matter, Reidel, Dordrecht.

Habing, H.J., Olnon, F.M., Chester, T., Gillett, F., Rowan-Robinson, M. \& Neugebauer, G., 1985. A $A p, 152, \mathrm{~L} 1$.

Haller, J.W. \& Rieke, M.J., 1989. In Morris, M., (ed) IAU Symp. 136, The Centre of the Galaxy, Reidel, Dordrecht.

Harmon, R. \& Gilmore, G., 1988. MNRAS, 235, 1025.

Hayakawa, S., Matsumoto, T., Murakami, H., Uyama, K., Thomas, J.A. \& Yamagami, T., 1981, A $A p, 100,116$.

Hiromoto, N., Maihara, T., Mizutani, K., Takami, H., Shibai, H., \& Okuda, H., 1984. A Ap, 139, 309.

Hofman, W., Lemke, D. \& Thum, C., 1977. A Ap, 57, 111.

Hofman, W., Lemke, D. \& Frey, A., 1978. A Ap, 70, 427. 
Ito, K., Matsumoto, T. \& Uyama, K., 1977. Nature, 265, 517.

Kent, S.M., Mink, D., Fazio, G., Koch, D., Melnick, G., Tardiff, A. \& Maxson, C., 1992. Ap J Suppl Ser, 78, 403.

te Lintel Hekkert, P., Caswell, J. \& Habing, H.J., A Ap, 1990.

Lloyd Evans, T., 1976. MNRAS, 174, 169.

Kinman, T.D., Feast, M.W. \& Lasker, B.M., 1988. $A J, 95,804$.

Landini, M., Natta, A., Oliva, E., Salinari, E. \& Moorwood, A.F.M., 1984. A Ap, 134, 284.

Maihara, T., Oda, N., Sugiyama, T \& Okuda, H., 1978. PASJ, 30, 1.

Matsumoto, T., Hayakawa, S., Koizumi, H., Murakami, H., Uyama, K., Yamagami, T. \& Thomas, J.A., 1982. In Riegler, G.R. \& Blandford, R.D., (eds) The Galactic Center, AIP Conference Ser. 83, Amer. Inst. Phys., New York, USA.

Menzies, J.W., 1990. In Jarvis, B.J \& Terndrup, D.M., Bulges of Galaxies, ESO Cnf \& Workshop Proc No 35.

Moneti, A., Glass, I.S. \& Moorwood, A.F.M., 1992. MNRAS, 258, 705.

Mould, J.R., 1992. In Barbuy, B. \& Renzini, A., (eds) IAU Symposium 149: The Stellar Populations of Galaxies, Reidel, Dordrecht.

Nakada,, Y., Deguchi, S., Hashimoto, O., Izumiura, H., Onaka, T., Sekiguchi, K. \& Yamamura, I., 1991, Nature, 353, 140.

Oda, N., Maihara, T., Sugiyama, T. \& Okuda, H. 1979. A Ap, 72, 309.

Okuda, , H., Maihara, T., Oda, N. \& Sugiyama, T., 1977. Nature, 265, 515.

Oosterhoff, P. Th. \& Ponsen, J., 1968. BAN Suppl Ser, 1, 397.

Rieke, G.H. \& Lebofsky, M.J., 1985. Ap J., 288, 618.

Rich, R.M., 1988. $A J, 95,828$.

Rich, R.M. \& Mould, J.R., 1991. AJ, 101, 1286.

Ruelas-Mayorga, R.A. \& Teague, P.F., 1992. A Ap Suppl., 93, 61.

Terndrup, D.M., Frogel, J.A. \& Whitford, A.E., 1990. Ap J, 357, 453.

Terndrup, D.M., Frogel, J.A. \& Whitford, A.E., 1991. Ap J, 378, 742.

van de Hulst, H.C., 1949. Rech astr Obs Utrecht, 11, pt 2.

van der Veen, W.E.C.J. \& Habing, H.J., 1988. A $A p, 194,125$.

van der Veen, W.E.C.J. \& Habing, H.J., 1990. A Ap 231, 404.

Walker, A., Sharples, R. \& Cropper, M., 1990. In Jarvis, B.J. \& Terndrup, D.M., (eds) Bulges of Galaxies, ESO Cnf \& Workshop Proc No 35.

Weinberg, M.D., 1992a. Ap J, 384, 81.

Weinberg, M.D., 1992b. Ap J, 392, L67.

Wesselink, Th., 1987. PhD Thesis, Nijmegen.

Whitelock, P.A. \& Catchpole, R.M., 1992. In Blitz, L., (ed) Large Scale Distribution of Gas and Dust in the Galaxy, Kluwer, Dordrecht.

Whitelock, P.A., Feast, M.W. \& Catchpole, R.M., 1991. MNRAS, 248, 276.

Wood, P.R. \& Bessell, M.S., 1983. Ap J, 265, 748.

\section{DISCUSSION}

Sellwood: I wonder what you mean by the Bulge. In particular, your conception that there seems to be a bar at the centre of the Galaxy. But you would not accept Weinberg's point of view, that there was nothing else. So what is it that you mean by the Bulge? Is it the bar, or is it something with additional components to that?

Glass: I would say that it is essentially a conglomeration that is dominated by the bar, but I don't except Weinberg's point of view that there is nothing else.

Sellwood: So the bar is a large part of it, but not all of it? 
Glass: I should imagine that it's a matter of which population you're looking at.

Feast: I think that the point of Weinberg is, that he comes to the conclusion that there is nothing that shows an $r^{1 / 4}$ distribution. But I think that's not new, there is practically nothing that shows an $r^{1 / 4}$ and that has been known for some while. If you say "You have to have an $\mathrm{r}^{1 / 4}$ distribution in order to have a bulge", then there's no bulge. But whatever the group of stars is, whether you think of it as a concentration of the disc or a concentration of the spheroid, there is in fact a large concentration of objects there and that in a way is the impcrtant thing for many studies. The rest tends to be rather a question of terminolog.\%.

Rich: The M31 luminosity function of field four: we in fact do confirm your Sagittarius field, we found exactly the cut-off at -5 . We've now extended the study to several other fields, as I will show later. We can disprove any evidence of disk contamination, there is a sharp cut-off of the luminosity function, just as you see. As we go in closer it goes up to -5.5 or so.

King: With regard to Sellwood's question: I think part of the answer is in the distinction I urge people to make about what population you're selecting your stars from. The metal rich population can't make Miras because it doesn't make an asymptotic giant branch.



At registration time: M. Schaetsaert, N. Baeck, J. Sellwood, (i. Bertin, S. Kent, H. Hasan 


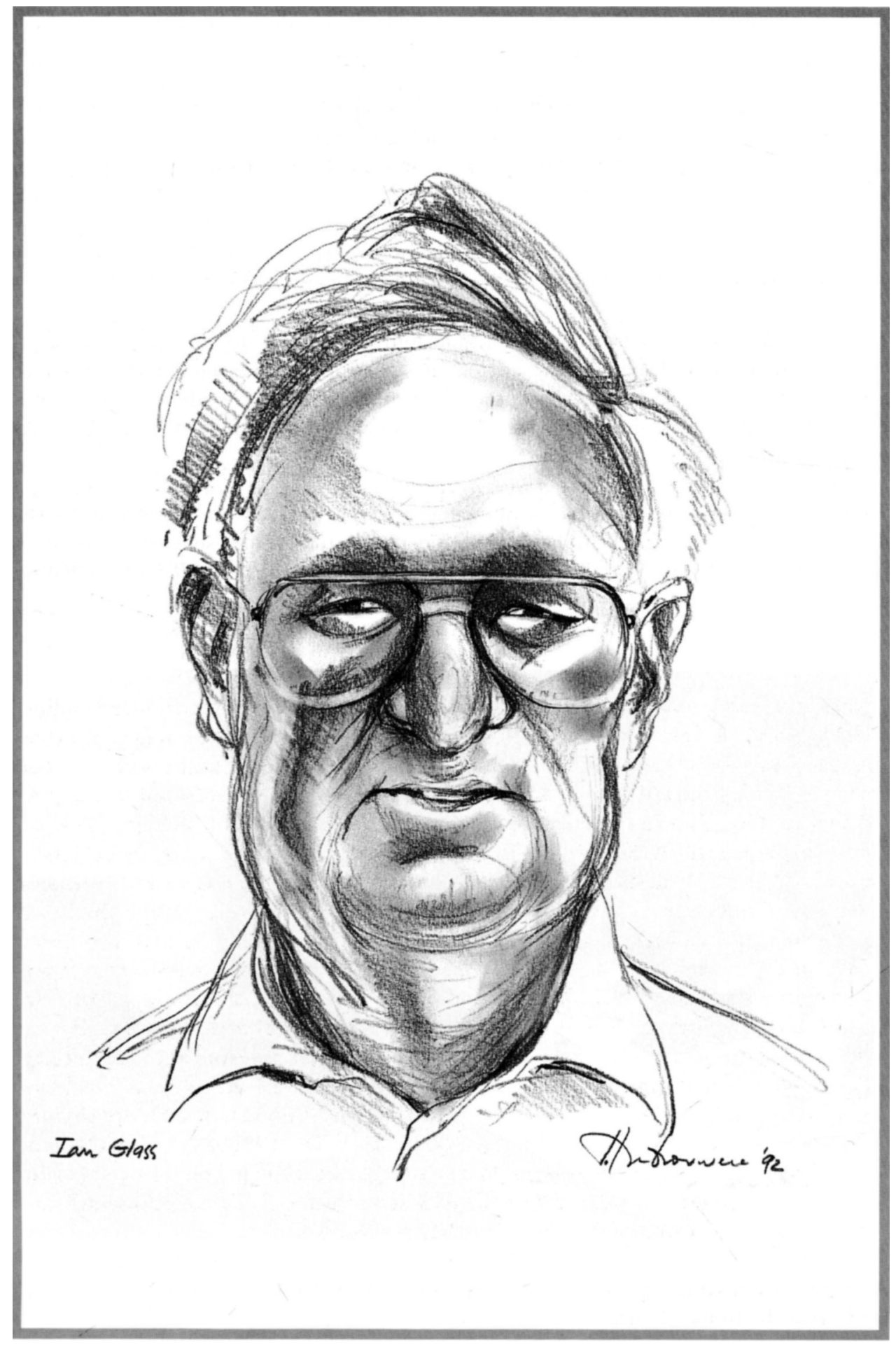

